\title{
Archéopages Archéopages
}

Archéologie et société

\section{Un camp d'internement de la seconde guerre mondiale près de Poitiers. Le Frontstalag 230 et le Centre de Séjour surveillé}

\section{Sonia Leconte}

\section{OpenEdition}

\section{Journals}

Édition électronique

URL : https://journals.openedition.org/archeopages/554

DOI : 10.4000/archeopages.554

ISSN : 2269-9872

Éditeur

INRAP - Institut national de recherches archéologiques préventives

\section{Édition imprimée}

Date de publication : 1 septembre 2014

Pagination : 76-81

ISSN : $1622-8545$

\section{Référence électronique}

Sonia Leconte, «Un camp d'internement de la seconde guerre mondiale près de Poitiers. Le Frontstalag 230 et le Centre de Séjour surveillé », Archéopages [En ligne], 39 | 10/2013-01/2014, mis en ligne le 01 janvier 2016, consulté le 22 janvier 2022. URL : http://journals.openedition.org/ archeopages/554 ; DOI : https://doi.org/10.4000/archeopages.554 


\title{
Un camp d'internement de la seconde guerre mondiale près de Poitiers Le Frontstalag 230 et le Centre de Séjour surveillé
}

\author{
Sonia Leconte ${ }^{\text {Irrap }}$
}

\section{6}

En périphérie ouest de Poitiers (Vienne), deux diagnostics archéologiques, menés en 2008 et $2011^{1}$ à la Chauvinerie, ont mis au jour les vestiges d'un camp d'internement ayant connu deux phases d'occupation (Leconte et al., 2008). En dehors des allées empierrées et des fossés périphériques, il ne reste plus aucune trace du camp. Les baraquements en bois, construits le plus souvent sur pilotis, n'ont laissé que peu d'indices, ce qui témoigne de la précarité de ces constructions, qui ont pu être facilement démantelées lors de la fermeture du camp. Seuls quelques foyers épars, des trous de poteau et des épandages de mobilier contemporain rendent compte de la vie du camp. Les investigations, qui n'ont pas donné lieu à la prescription d'une fouille, ont cependant permis la redécouverte de ce site que la mémoire collective, malgré l'existence d'une documentation archivistique relativement abondante, avait préféré occulter.

À la suite de la défaite française, les Allemands réquisitionnent la caserne Ladmirault et y installent le Frontstalag 230 le 20 juillet 1940. À la différence des prisonniers de métropole transférés en Allemagne, les combattants coloniaux - Antillais,
Malgaches, Indochinois, Sénégalais, Marocains, Tunisiens -, interdits de séjour sur le sol allemand, y sont placés sous l'autorité militaire allemande. Le camp, qui regroupe plus de 7300 détenus, est fermé en février 1942 et repasse aux mains de l'État français.

Il est réouvert par les autorités françaises, de février à novembre 1945. Il comprend alors un Centre de Séjour surveillé (CSS), placé sous la tutelle du Ministère de l'Intérieur, ainsi qu'un camp, ou dépôt $n^{\circ}$ 91, de prisonniers de guerre de l'Axe (PGA). Le CSS est réservé aux civils : hommes, vieillards, femmes et enfants. Parmi eux, des ouvriers de la Sarre allemande travaillant en Sarre française, des Alsaciens ayant eu une attitude jugée pro-allemande, des personnes déplacées en attente d'une libération. Le premier convoi de civils (février 1945) comprend 952 personnes dont 128 femmes et 180 enfants. Les convois s'accélèrent rapidement : en juin, l'effectif est porté à 3700 personnes. Le dépôt $n^{\circ} 91$ regroupe, quant à lui, environ 7000 prisonniers de guerre dont 4 ooo Allemands et 3 zooo Hongrois.
1. Les diagnostics ont été menés à Poitiers, ZAC des Montgorges, sous la direction de Sonia Leconte : phase 1 mai 2008 , phase 2 : juin 2011. L'extrémité septentrionale du site est désormais affectée au Régiment d'Infanterie chars de marine (RICM) 

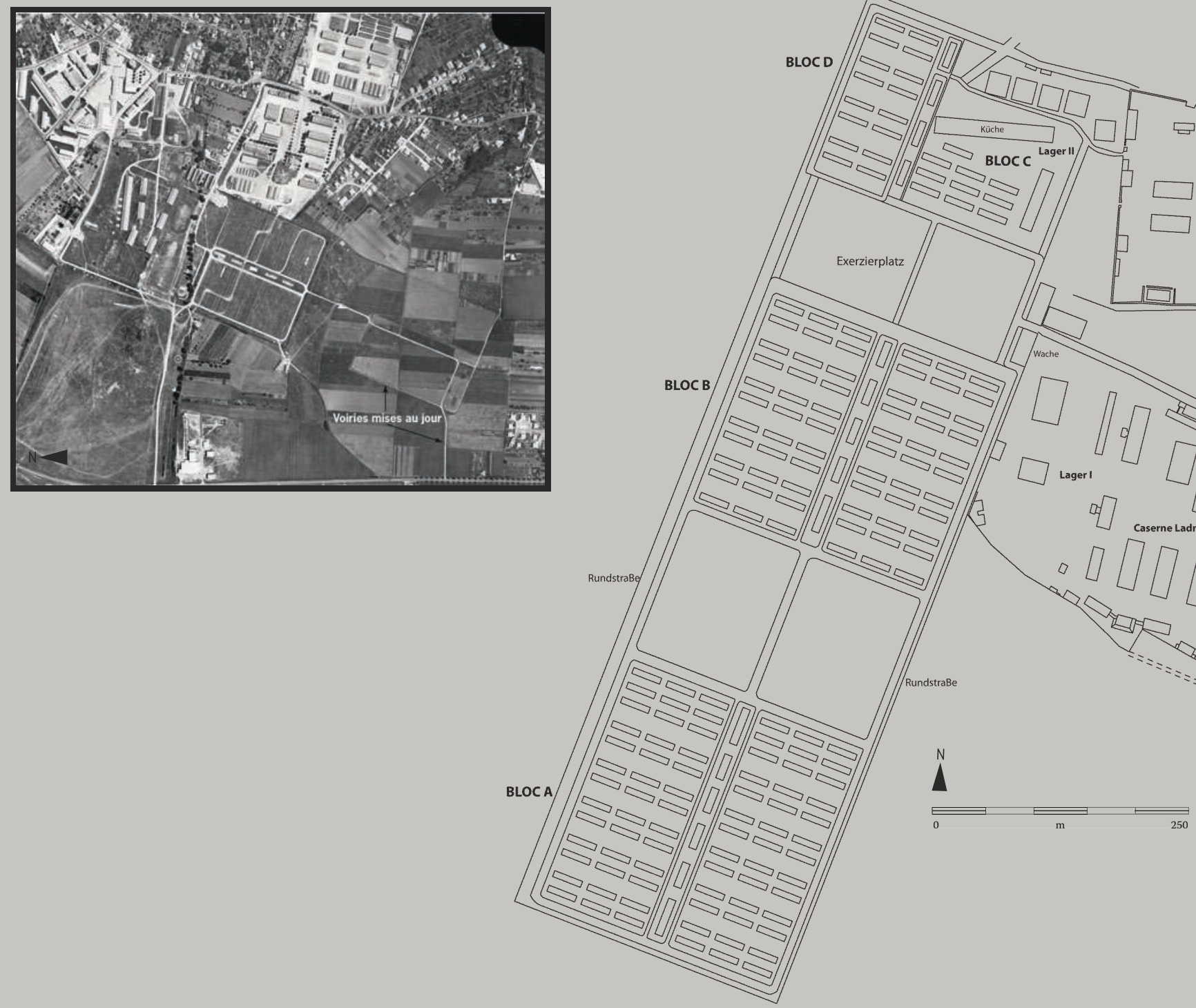

Les plans des locaux

Les locaux de la caserne Ladmirault, réquisitionnés par les Allemands en 1940, s'avérant très rapidement insuffisants pour accueillir tous les prisonniers, ces derniers sont mis à contribution pour édifier ex nihilo, d'octobre à décembre 1940, quatre blocs de baraquements, portant la capacité d'accueil du camp à plus de 9000 personnes. Le recours aux photographies aériennes et aux archives a permis de restituer de vastes quadrilatères entourés de fils de fer barbelés sur plusieurs rangées. Un rapport de 1944, dressé en prévision de la réutilisation du camp, permet de reconstituer son organisation. La première enceinte (bloc C sud) dispose d'un grand bâtiment maçonné pouvant loger 300 prisonniers, servir de réfectoire ou être aménagé en infirmerie, de 10 baraques pouvant contenir chacune 100 prisonniers, d'un bâtiment en briques contenant les WC et d'un bâtiment en maçonnerie de grandes dimensions dans lequel se trouvent les cuisines et le magasin aux vivres. La deuxième enceinte (bloc $D$ avant agrandissement) compte 9 baraques ( 100 prisonniers par baraque). La troisième 3 (bloc $C$ nord) regroupe 4 baraques pouvant accueillir 300 personnes ainsi qu'une baraque avec WC. La quatrième (bloc B) comprend 66 baraques. $\AA$ côté de ces aménagements, le dernier bloc (A) comprend 78 baraques. Le plan orthogonal semble avoir été systématiquement utilisé dans le cas des créations ex nihilo. Les formes de l'internement se voulaient ainsi rationalisées et fonctionnelles, à même d'établir et de faciliter la gestion du personnel et des prisonniers. Cette organisation permettait une répartition des effectifs et une séparation catégorielle des populations présentes au sein du camp. Les structures administratives et les organes décisionnels étaient relégués en périphérie, tandis que chaque îlot était scindé en deux ensembles distincts d'une capacité équivalente. Le Centre de Séjour surveillé comprenait ainsi 33 baraques réservées aux hommes et 33 baraques réservées aux femmes et aux enfants de moins de 14 ans, la séparation étant matérialisée dans l'espace par des installations sanitaires communes, sises au centre de l'îlot (bloc B). 


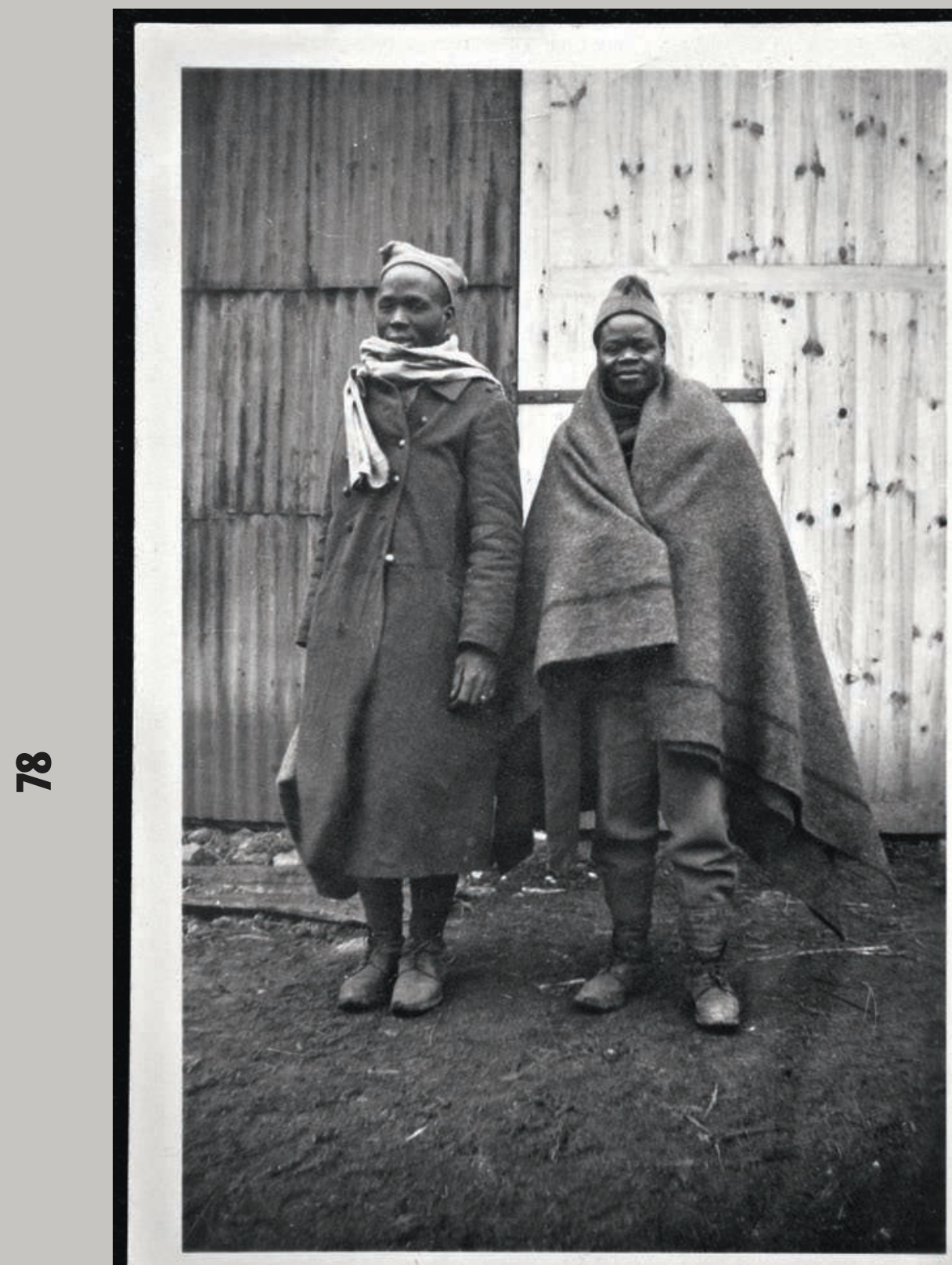

La prière est tolérée, tandis que le souci de la santé morale des prisonniers s'illustre par le recours aux jeux (dominos ou dés, très prisés des armées), aux livres (distribués ponctuellement par la Croix-Rouge et les Amitiés Africaines); des cours sont également dispensés au sein du camp. La réception de colis et de lettres est permise. Des marraines de guerre, recrutées parmi la population française, notamment des élèves de 13-15 ans, écrivent aux détenus et tentent d'entretenir leur moral. Une couverture est fournie à chaque détenu. La CroixRouge distribue des vêtements usagés; gants et chaussettes manquent cruellement, ce qui cause des engelures. Les rations alimentaires, surtout composées de

rutabagas et de pommes de terre, sont complétées par des colis réguliers d'associations.
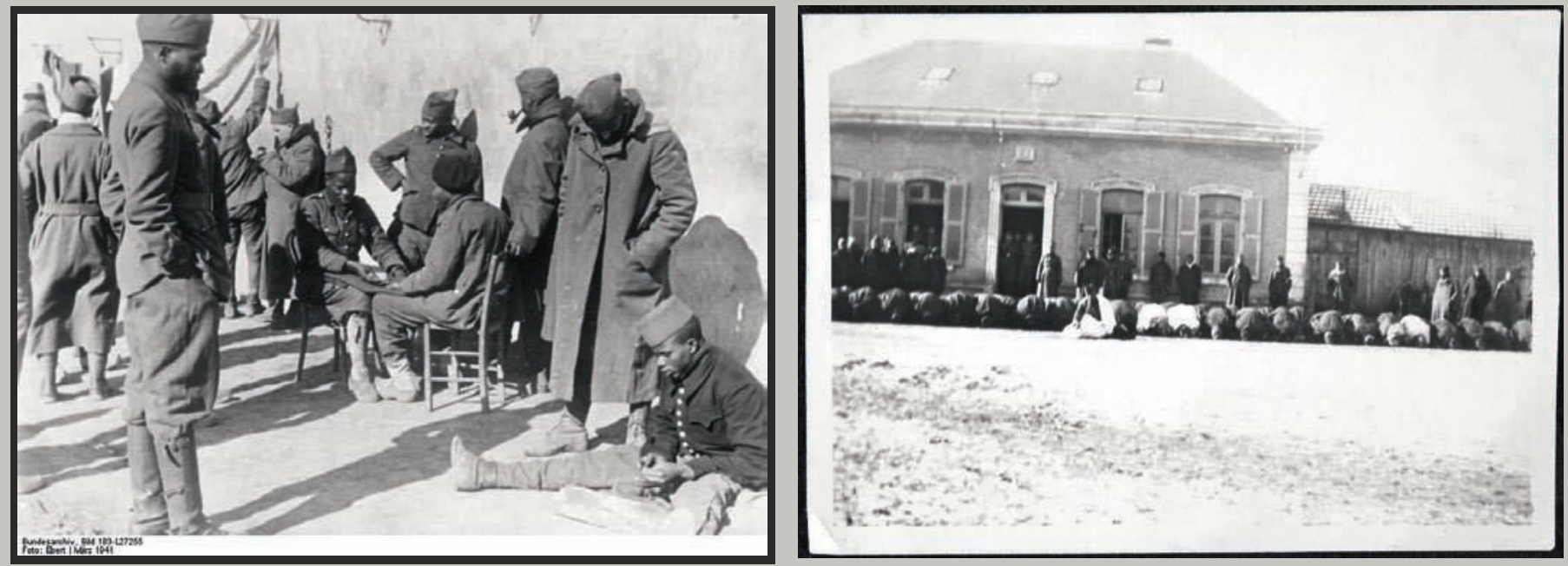
Les conditions de détention au.

\section{Frontstalag 230}

Les conditions de détention des troupes coloniales sont documentées par le témoignage écrit de Léopold Sédar Senghor, détenu d'octobre à novembre 1941 au Frontstalag, document inédit découvert en 2010 par l'historien allemand Raffael Scheck (professeur au Colby College - Waterville, Maine). Senghor y décrit le froid, le manque d'installations sanitaires, le développement de maladies - notamment la tuberculose - et les tensions entretenues par les Allemands entre les différentes nationalités.
Les archives confirment ce constat : de janvier à avril 194 1, 30 décès sont dus à la tuberculose ; toutes causes confondues, 76 individus meurent au mois d'avril. Les photographies témoignent cependant aussi du respect de quelques principes émis par la convention de Genève (1929). Par ailleurs, les relations avec l'autorité et, en premier lieu, avec les gardiens n'apparaissent pas toujours hostiles et inamicales. Des cas de fraternisation ont été observés. Senghor lui-même noue une relation d'amitié avec son gardien, linguiste de formation, qui perdurera après la guerre.

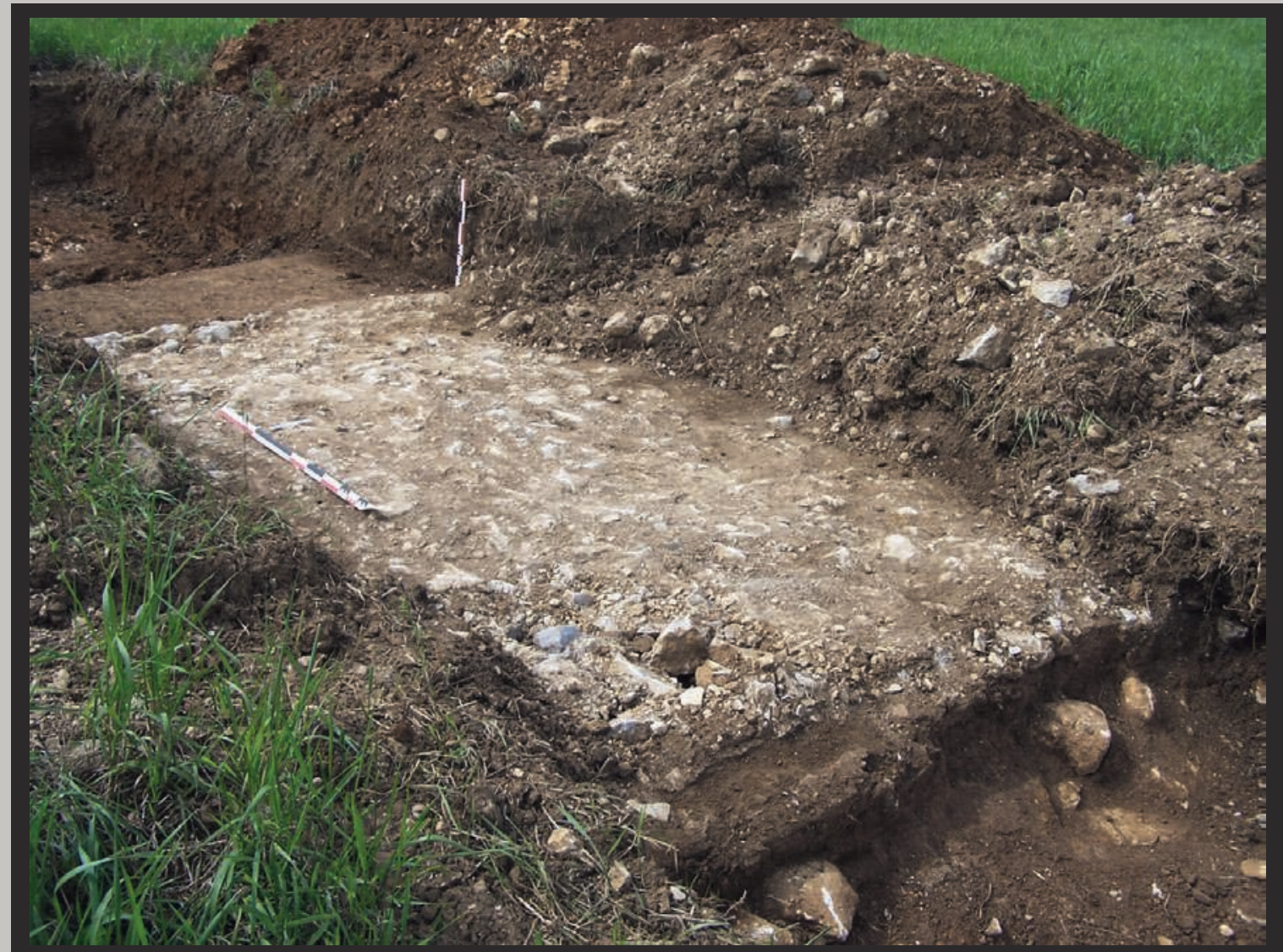

Les allées empierrées, apparues, lors du diagnostic, directement sous la terre végétale largeur conservée de $4 \mathrm{~m}$ en moyenne pour une quarantaine de centimètres

d'épaisseur ont permis l'assainissement du terrain ; les blocs de calcaire gréseux qui les constituent ont été compactés par les détenus à l'aide de rouleaux à main. 


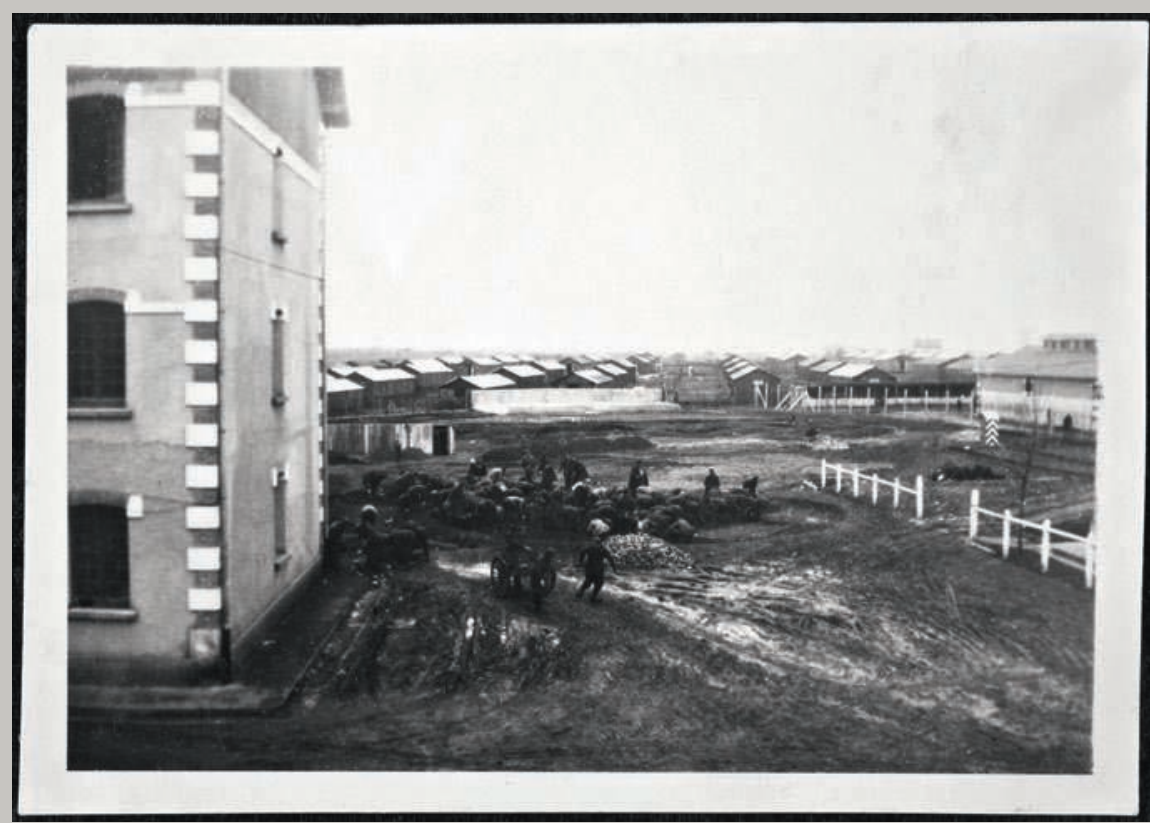

Cette photographie du CSS montre les captifs au travail. S'assurer de labonnesantémorale desprisonniersde guerre est un devoir qui incombe à tout commandant de camp, selon la convention de Genève. L'oisiveté est combattue.Letravailest organiséà l'intérieur du camp sous la forme de corvées diverses (nettoyage, assainissement, etc.). De plus, ces personnes provenant de tous les milieux socio-

professionnels sont

très vite perçues par

l'État comme une

manne économique.
Sur la base du

volontariat, les

prisonniers sont employés à l'extérieur, que ce soit dans l'agriculture ou à la reconstruction du pays (au déblaiement des zones bombardées de Poitiers, aux usines, à la gare). Cela concerne avant tout les hommes, seules quelques femmes sortent du camp pour effectuer des travaux de couture et de lessivage.

\section{Les conditions de détention}

\section{à la Chauvinerie en 1945}

À la lecture des archives, les conditions de détention dans le Centre de Séjour surveillé en 1945 -documentées par les Inspections du Comité International de la Croix-Rouge et par la correspondance du préfet de la Vienne avec le commissaire de la République - paraissent catastrophiques. Le taux de mortalité y est sept fois plus important que dans les autres camps du même type. La situation est particulièrement grave chez les enfants, le camp étant totalement inadapté. Fn avril 1945, lors de la promulgation du règlement intérieur du camp, la construction d'une pouponnière est prévue. Il y a alors 102 enfants de moins de 3 ans. Dans les faits, la pouponnière ne sera jamais totalement achevée. Pas un des 64 enfants nés en captivité ne survivra. Les alertes de la CroixRouge se voient confirmées lors de l'été caniculaire de 1945 , qui ne fait qu'augmenter le nombre des décès. Fn août 1945 est dévoilé ce que l'on a appelé le « scandale du camp ». Des gardiens témoignent qu'aux difficultés matérielles se sont ajoutées des exactions. L'enquête judiciaire ouverte en septembre 1945 pour " vol au préjudice des internés et de l'ítat » établit plusieurs délits. L'argent et les effets personnels des captifs, confisqués à leur entrée dans le camp, étaient spoliés. Les gestionnaires ainsi que certains gardiens détournaient une partie des aliments d'où des rations extrêmement chiches : une soupe de carotte pour tout repas et une boule de pain pour trois personnes. Les crédits alloués à la nourriture étaient partiellement détournés. Des maraîchers complices livraient des produits avariés et acceptaient de fausses factures. Le lait destiné aux enfants et aux malades était également prélevé sur les rations, à raison de 3 litres par jour, et reversé au directeur. D'autre part, le directeur du camp avait réalisé des travaux sanitaires à son domicile, travaux imputés sur les dépenses générales du camp. Cette affaire connaît un grand retentissement dans la presse. Pourtant les condamnations sont maigres. Les gestionnaires sont amnistiés, tandis que le directeur du camp est suspendu et remplacé.

Le scandale précipite la fermeture du camp, le $1^{\text {er }}$ novembre 1945. Les détenus sont redirigés vers d'autres camps français. Les baraquements et cantonnements, afférents au CSS, sont réutilisés pour les prisonniers de guerre de l'Axe, le camp voisin étant devenu nettement insuffisant pour tous les captifs qu'il renferme. Leur sort semble tout aussi dramatique : un article de presse de la Nouvelle République, daté du 23 octobre 1945, évoque de "vrais fantômes flottant dans des capotes kakis ", soumis à un régime de sous-alimentation, une mortalité « effrayante ", des jeunes mourant d'inanition et " pas de paquets, pas de lettres ». La population ne semble pas s'en être émue ou souciée : la France était exsangue, les Allemands étaient les responsables. Ce passé douloureux a contribué à faire disparaître de la mémoire collective l'existence de ces camps que l'archéologie permet de redécouvrir. 


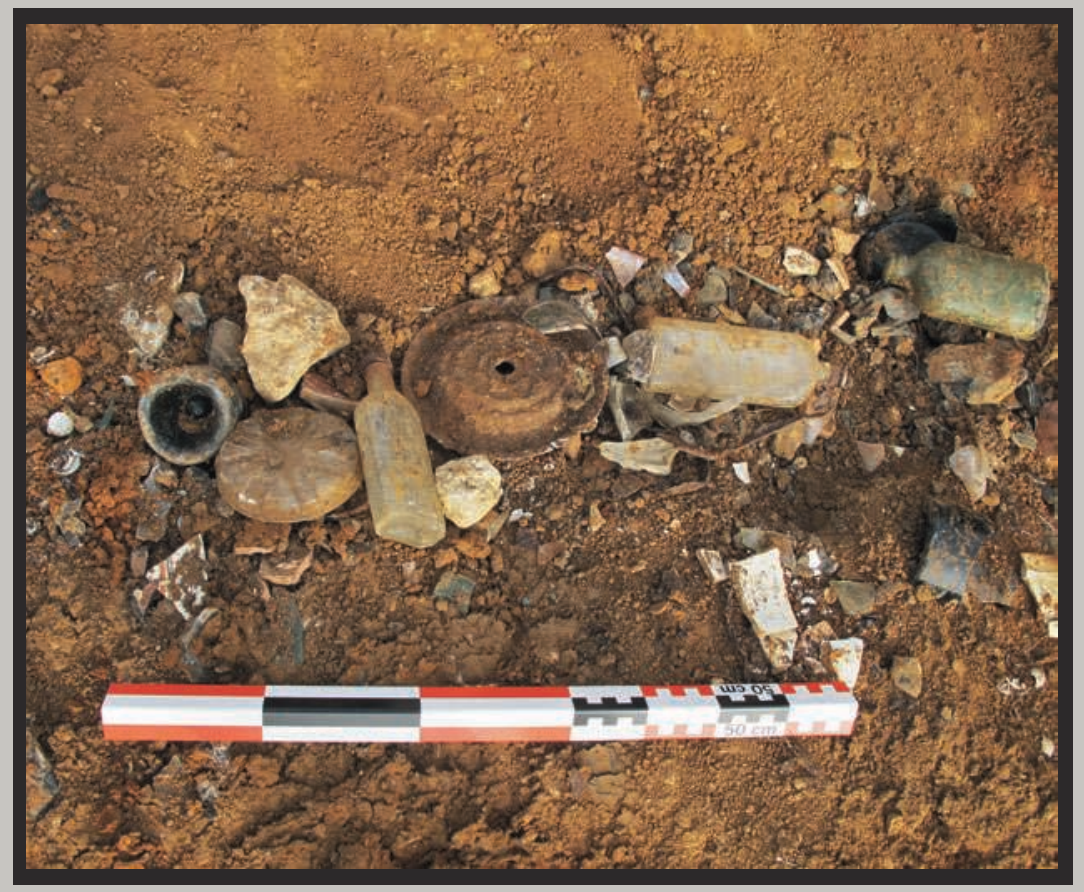

Références bibliographiques

HIERNARD J., LECONTE S., à paraître « La Chauvinerie : un camp sorti de l'oubli », Revue historique du Centre-Ouest, $2^{\mathrm{e}}$ semestre 2014.

Leconte S., Bernard R., Coutureau M., Bambagioni F, 2008, Poitiers (Vienne), ZAC des Montgorges IA et $I B$, rapport de diagnostic, Inrap-SRA PoitouCharentes, $38 \mathrm{p}$.

LeConte S., Bambagioni F., Bernard R., Connet N. 2011, Poitiers (Vienne), ZAC des Montgorges, phase 2, rapport de diagnostic, Inrap-SRA Poitou-Charentes, 59 p.
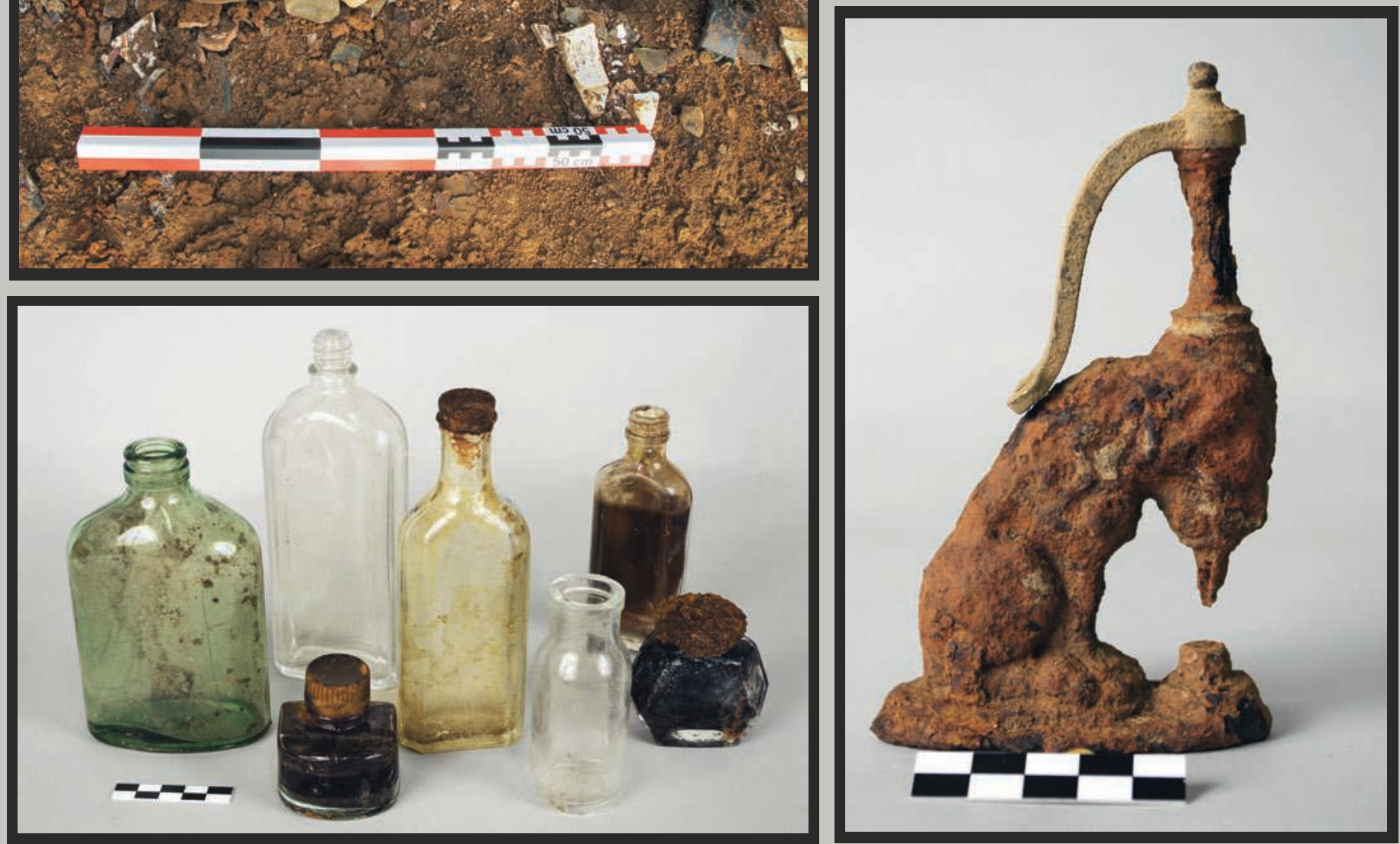

La présence de fioles médicamenteuses d'un contenu indéterminé, ainsi que celle d'un flacon des Laboratoires Fosse, inventeurs du Livartil, préconisé dans le traitement des affections hépatiques, témoignent de la sous-alimentation, àl'origine de nombreuses carences. Alors que les ustensiles alimentaires sont principalement constitués par des récipients métalliques (conserves, gamelles), l'opération archéologique a mis au jour une importante part de vaisselle en faïence. Était-elle uniquement réservée aux détenus civils des camps? A-t-elle été fournie à l'occasion d'échanges et de trocs avec la population extérieure? Parmi les artefacts découverts, un objet insolite (ci-dessus, photographie à droite) a été recueilli : un pose œillet en fonte. Celui-ci présente une forme de lion assis, dont la gueule accueille un poinçon.Associé au travail du cuir

(cordonnerie) ou à celui de la couture, il illustre parfaitement les tâches de la vie quotidienne, les internés étant amenés à rapiécer leurs chaussures et leurs vêtements dégradés. Les encriers retrouvés au cours de l'opération témoignent de l'importance de l'écriture, activité primordiale et nécessaire : raconter, s'éloigner symboliquement des lieux et de sa condition, garder le lien avec les siens, avec l'extérieur, en attendant une libération prochaine. Selon les instructions, les internés pouvaient envoyer et recevoir deux lettres et deux cartes par mois ainsi que quatre colis de $5 \mathrm{~kg}$ chacun par mois. Trois à quatre interprètes allemands censuraient les correspondances et vérifiaient les colis. 